\title{
MODELING \& SIMULATION OF SOFTWARE ARCHITECTURES OF SYSTEMS-OF-SYSTEMS : AN INDUSTRIAL REPORT ON THE BRAZILIAN SPACE SYSTEM
}

\author{
Valdemar Vicente Graciano Neto \\ Federal University of Goiás \\ Goiânia, Goiás, Brazil \\ valdemarneto@inf.ufg.br
}

Adair José Rohling

Federal Technological University of Paraná

Guarapuava, Brazil

adairrohling@utfpr.edu.br

\author{
Carlos Eduardo Paes \\ Pontifical Catholic University of São Paulo \\ São Paulo, Brazil \\ carlosp@pucsp.br \\ Wallace Manzano \\ Elisa Yumi Nakagawa \\ University of São Paulo \\ São Carlos, Brazil \\ wallace.manzano@usp.br, elisa@icmc.usp.br
}

\begin{abstract}
Systems-of-Systems (SoS) have demanded for novel solutions to deal with their large-scale, dynamic, and critical nature. Then, software engineering and simulation communities have been motivated to develop solutions to engineering such systems. In this direction, this paper presents an industrial experience report of a modeling and simulation $(\mathrm{M} \& S$ ) of a Space SoS, which was conceived in the Brazilian National Institute for Space Research (INPE) and is composed of multiple satellites, data collection platforms, and information systems. We used MS4ME platform and enriched it with a model transformation approach (from a formal architectural descrition to DEVS models). We hope this paper can foster discussions towards unification of efforts from both communities.
\end{abstract}

Keywords: model-driven, model-based, system-of-systems, simulation, industry.

\section{INTRODUCTION}

Systems-of-Systems (SoS) are a type of complex systems. SoS constituent systems have received an increasing portion of software to improve their operational accuracy and allow them to interoperate. Examples include traffic radars, sensors, houses, and hospitals, which have become smarter by receiving software. Several constituents are IoT-based systems, software-intensive information systems or even other SoS. Currently, SoS often support critical domains, such as emergence and crisis response systems (Graciano Neto, Garcés, Guessi, Paes, Manzano, Oquendo, and Nakagawa 2018). Hence, if they do not work with precision, their users can face great danger (Zeigler, Muzy, and Kofman 2019), which makes it very risky to develop them from scratch, and with no quality assurance process still at design-time. Therefore, simulation models have been used to advance and predict, at design-time, the structure and behavior of SoS, in order to minimize failures that could be fatal after they are put into operation. 
In parallel, due to the high amount of software that has been embedded in the SoS constituents, an area has emerged, namely Software Engineering for SoS (SESoS), which addresses principles of software engineering for creating software systems that will be part of a SoS. In this context, quality concerns become critical, such as interoperability, availability, resilience, security, and fault tolerance. In software engineering, the concept of software architecture covers the quality requirements and how they are offered by a SoS. And when considered a SoS, its software architecture involves all the constituents' software elements, as well as the environment around them and the means used for their interoperability (Nielsen, Larsen, Fitzgerald, Woodcock, and Peleska 2015).

Thus, simulating SoS software architectures poses some additional concerns over simulation of single systems, such as: (i) use of a high level of abstraction language, since software engineers do not deal well with low-level languages of abstraction; (ii) formal basis for verification and formal validation, given the domain's criticality; (iii) reliable representation and prediction of relevant quality attributes; (iv) precise representation of the structure and behavior (Taylor, Medvidovic, and Dashofy 2010) of the constituents and their interoperability; and (v) representation of the SoS surrounding environment. However, models to be simulated in software engineering are usually static (not dynamic), and unsuccessful to represent both the static and dynamic structure and behavior of a SoS. This difficulty is further aggravated when we think of the representation of the dynamics between multiple systems. Despite the existing trends such as models at runtime (Blair, Bencomo, and France 2009), and executable versions of UML (OMG 2015) and SySML (OMG 2019) languages, none of these languages have been very successful in representing interoperability between multiple systems that maintain their operational and managerial independence.

In this sense, in this paper we present a contribution in systems modeling and simulation (M\&S), reporting how both paradigms were harmonized to meet all the requirements stated above. We used our toolset and research results to model and simulate a software architecture of a Space SoS, an indispensable sector to any country. The paper is structure as follows: Section 2 presents the foundations necessary to understand some technical aspects of the solution; Section 3 provides a retrospective of our research advances and briefly reports the application of our solutions in the Brazilian Space SoS context; Section 4 presents a discussion of our results and established a parallel between the advances of simulation community and software engineering for SoS community, besides providing a list of open gaps that shall still be bridged as a joint effort of both communities; and Section 5 concludes the paper with final remarks.

\section{MODELING AND SIMULATION OF SYSTEMS-OF-SYSTEMS SOFTWARE ARCHITECTURES}

Systems-of-Systems (SoS) have become software-intensive, which means that software essentially influences their design, construction, deployment, and evolution (ISO 2011, Goncalves, Cavalcante, Batista, Oquendo, and Nakagawa 2014). Examples of SoS include smart cities, emergence and crisis response management systems, space systems, ambient assisted living, and even constituents of smart cities are themselves SoS, such as smart buildings, smart traffic control, and smart homes (Graciano Neto 2018, Cavalcante, Cacho, Lopes, and Batista 2017). SoS are characterized by well-defined dimensions, which include operational and managerial independence of constituent systems, evolutionary development, distribution, and emergent behaviors (Maier 1998). Heterogeneity and hierarchical organization are also considered SoS characteristics (Boardman and Sauser 2006, Lana, Souza, Delamaro, Nakagawa, Oquendo, and Maldonado 2016). Moreover, dynamic architecture characteristic is a consequence of operational and managerial independence of constituents, which causes changes to the SoS architectural arrangement due to constituents that join or leave the SoS at runtime (Oquendo 2016a, Manzano, Graciano Neto, and Nakagawa 2018). Since SoS are a type of complex systems, complexity is another dimension, as it involves a large number of constituents and connections among them (INCOSE 2018, Yingchao 2012). 
Since software intensely impacts SoS and has become an intrinsic part of it, SoS currently has a softwareintensive architecture. Herein, it is important to distinguish between software architecture and system architecture. From the Systems Engineering perspective, a system refers to a combination of elements that function together to produce the capability required to meet a need, including all hardware, software, equipment, facilities, personnel, processes, and procedures needed for this purpose (Shea 2017). Then, a systems engineer is concerned with a holistic view of all elements of a system, including both hardware and software and, more particularly, all of the interfaces of the device (INCOSE 2018). Hence, a system architecture comprises (i) the programmable hardware artifact, or computing machine, which materializes the physical device that will host and run software; (ii) software artifacts, which comprise the logical part that will run over a (hardware) physical machine and control its operation; and (iii) all the other relevant elements, including the surrounding environment, people, information, and processes.

In turn, a software architecture involves the (i) software elements, relations among them, properties of both elements, and of the surrounding environment (Garlan, Bachmann, Ivers, Stafford, Bass, Clements, and Merson 2010), and (ii) the documentation of the fundamental design decisions and rationale, besides patterns, business rules, and description of their behaviors (Taylor, Medvidovic, and Dashofy 2010, ISO 2011). Then, a SoS software architecture involves the software part of the SoS constituent systems, the logical connectors (including middleware to abstract heterogeneities and distributed software to support services and interoperability), the properties of the constituents (their business rules, owners, and requirements), and the surrounding environment (Nielsen, Larsen, Fitzgerald, Woodcock, and Peleska 2015). Software architectures are highly influenced by quality attributes, which are non-functional properties that must be achieved to increase the quality of the final product, such as availability, performance, security, and usability (Bass, Clements, and Kazman 2012). Software architectures are often specified using architectural description languages (ADL), which are special types of domain-specific languages (DSL) particularly conceived to precisely capture software architectures (Guessi, Graciano Neto, Bianchi, Felizardo, Oquendo, and Nakagawa 2015, Medvidovic and Taylor 2000). An example of a language that documents software architecture specifications is SoSADL (Oquendo 2016a).

Modeling and Simulation $(M \& S)$ is an ascending paradigm for SoS. It comprises the combined adoption of model and simulation to represent both structure and behavior of a SoS. DEVS (Discrete EVent System specification) is a remarkable instance of a language that is often used for M\&S purposes in SoS domain. Models capture the relevant concerns of system of interest to be developed for a particular domain, whilst simulation enables the prediction of its behaviors. We can establish a parallel between SoSADL and DEVS, which are the state of the art languages to represent SoS software architecture and SoS system architecture, respectively. Table 1 brings such comparison. If we recall the aforementioned parameters that are relevant to simulate SoS software architecture, we observe they are, actually, complementary. Despite being abstract and enabling SoS engineers to specify state machines with precision and using natural language (DEVSNL), specifying constituents in DEVS requires to use notion of ports, and to specify state machines, which are quite low-level of abstraction and error-prone. Both languages present formal foundation, which enables verification and validation $(\mathrm{V} \& \mathrm{~V})$ procedures. Despite supporting the representation of quality attributes, none of the languages natively provides language constructs to explicitly represent quality attributes, which are prominent for quality assurance of SoS software architectures. SoSADL provides a construct called environment, which enables the architect to specify a constituent connection as that one that senses data from the environment. Both allow the static specification of SoS considering its parts, but only DEVS allows the execution of its models, providing a dynamic view of the SoS behaviors still at design-time.

We then decided to harmonize both formalisms to achieve the requirements for simulation of a SoS software architecture mentioned in Table 1. We combined them using a Model-Based Software Engineering (MBSE) approach, establishing a model transformation between them, which is a mapping between the considered models that establishes a traceability link between them, enabling an automatic conversion of one formalism into another one (Graciano Neto, Manzano, Kassab, and Nakagawa 2018). Models in SoSADL (Oquendo 
Table 1: A parallel between SoSADL and DEVS in regards to requirements for simulation SoS software architectures.

\begin{tabular}{|l|c|c|}
\hline Parameter & SoSADL & DEVS \\
\hline Use of a high level of abstraction language & $\mathrm{x}$ & \\
\hline Formal foundation & $\mathrm{x}$ & $\mathrm{x}$ \\
\hline Representation of quality attributes & & \\
\hline Representation of the SoS surrounding environment & $\mathrm{x}$ & \\
\hline Static models & $\mathrm{x}$ & $\mathrm{x}$ \\
\hline Dynamic models & & $\mathrm{x}$ \\
\hline
\end{tabular}

2016a, Oquendo 2016c) were used as input for a model transformer, which produced DEVS models to be deployed and run in MS4ME platform (Zeigler, Sarjoughian, Duboz, and Souli 2012).

Studies on M\&S for software architecture are not abundant, but they are not exactly a new trend. Bogado et al. have worked on the modeling and simulation of software architectures, considering quality attributes and a few metrics (Bogado, Gonnet, and Leone 2014). However, their approach covers only single systems, not SoS, as ours. Akshay Rajhans and his colleagues have considered simulations as architectural views of an underlying base architecture of a cyber-physical system (Rajhans, Bhave, Loos, Krogh, Platzer, and Garlan 2011, Rajhans, Bhave, Ruchkin, Krogh, Garlan, Platzer, and Schmerl 2014). Nevertheless, their studies have not been conducted in SoS domain. Next section details the advances we achieved and the industrial case study.

\section{MODELING AND SIMULATING THE BRAZILIAN SPACE SOS}

This section details a retrospective on the advances achieved, and the industrial case on the Brazilian Space SoS.

A retrospective on the advances achieved. Recently, we have reported our advances (Graciano Neto, Manzano, Kassab, and Nakagawa 2018). We have worked in the last five years to build advances and foundations to support the modeling, simulation, and analysis of SoS software architectures. After reviewing the literature, we have perceived that, although classic languages were being used to specify SoS, they did not cope with all the important concerns of a SoS that should be addressed to make it reliable. Results revealed that several languages have been adopted in MBSE solutions for SoS engineering (Graciano Neto, Guessi, Oliveira, Oquendo, and Nakagawa 2014, Guessi, Graciano Neto, Bianchi, Felizardo, Oquendo, and Nakagawa 2015, Lana, Souza, Delamaro, Nakagawa, Oquendo, and Maldonado 2016). General purpose languages (such as UML, SySML, and others) were being used to specify SoS (Guessi, Graciano Neto, Bianchi, Felizardo, Oquendo, and Nakagawa 2015, Klein and van Vliet 2013). However, they have lacked essential constructs to represent all the relevant aspects of a SoS (Guessi, Cavalcante, and Oliveira 2015). On the other hand, simulations have been used to represent SoS dynamics (Dobrica and Niemele 2002, Michael, Riehle, and Shing 2009). However, none of them were able to cope with the aforementioned requirements, in particular to support the prediction of dynamic architectures impact on the SoS runtime execution.

Then, SoSADL emerged. It comprised an ADL formally founded on $\pi$-calculus that offered a syntax for specification in high-level of abstraction (Oquendo 2016a, Oquendo 2016b, Oquendo 2016c). However, despite supporting the representation of SoS dynamics, the language was still static. Then, we surveyed the literature to find some notation that could be appropriate to deal with SoS peculiarities, specially supporting dynamic reconfiguration due to operational independence, multiple and heterogeneous constituents, support to environmental modeling and simulation, representation of dynamics of SoS with an specification of the interoperability between constituents, that is, the data exchanged among them, and a type of time represen- 
tation to be based upon (Graciano Neto, Manzano, Kassab, and Nakagawa 2018). Then, we opted by DEVS as the formalism to simulate SoS software architectures (Zeigler, Sarjoughian, Duboz, and Souli 2012).

We have established a model transformation that maps SoSADL models into DEVS models. Such combination enabled us to achieve most of the requirements mentioned in Table 1. We also established some other important advances, which were (Graciano Neto 2018, Graciano Neto, Manzano, Kassab, and Nakagawa 2018):

Stimuli-SoS: Environment representation and stimuli generators. To represent the surrounding environment during the SoS software architecture simulation, we have established Stimuli-SoS. This approach automatically reads the SoSADL architectural description models and produce stimuli generators, i.e., artificial entities to continuously deliver data to feed the SoS simulation and imitate the SoS surrounding environment, automating the stimuli input (Yang, Wille, Grobe, and Drechler 2012, Piccolboni and Pravadelli 2014). This automated the execution of the SoS simulation, dispensing the need for the presence of a human during the simulation (Graciano Neto, Paes, Garcés, Guessi, Oquendo, and Nakagawa 2017);

Dynamic-SoS to represent SoS dynamic architectures. We also implemented the automatic generation of an artificial dynamic reconfiguration controller (DRC). This entity is automatically generated by the model transformation, and linked to all the constituents in the SoS architecture, deployed together with the simulation to perform changes during the simulation, enabling the prediction of the impact of the change of the functionalities being provided by the SoS (Manzano, Graciano Neto, and Nakagawa 2018);

Back-SoS method. Due to the inherent dynamics of SoS architectures, the runtime architectures frequently become outdated regarding the original architectural description. Due to this phenomenon, we also established Back-SoS, a backward model transformation approach that reestablishes the conformance between the simulation model at a given time and the SoSADL architectural description, updating the original model (Graciano Neto, Manzano, Garcés, Guessi, Oliveira, Volpato, and Nakagawa 2018); and

ASAS evaluation method. On top of such SosADL2DEVS model transformation and automatic generation of stimuli generators, we established an evaluation method based on simulations so-named ASAS

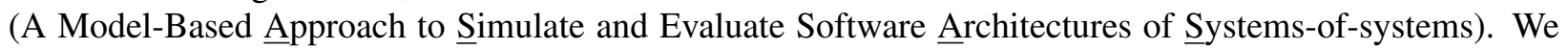
established a method composed of a well-defined set of steps to support the evaluation of SoS architecture behaviors based on a set of pre-defined attributes (Graciano Neto, Garcés, Guessi, Paes, Manzano, Oquendo, and Nakagawa 2018).

A report of an industrial application. Space activities have become essential to the daily life of every nation on Earth. The space industry provides increasingly more and better solutions, products and services. Space activities revenue exceeds US\$280 billion a year worldwide. In Brazil, the required annual investment is in the order of US\$ 250 million up to 2021, allocated to satellite mission projects, space access projects, space infrastructure and to other special and complementary projects (Brazilian Space Agency 2018). Brazil has over 13 million $\mathrm{km}^{2}$ of territory, rich in natural resources of all kinds, which needs to be increasingly identified, studied, monitored, managed, explored and protected. Space systems are vital to this effort and the space industry has an important role to fulfill.

Space systems are essential to any country. They offer the means (in particular satellites) to provide telecommunications, monitoring of natural resources and environmental or climatic changes, more speed and competence to face natural disasters, more vigilance at sea borders and coasts, reduction of regional inequalities, more promotion of social inclusion. All of this requires more space systems, launchers and launches, satellites, information and images coming from space, and space activities.

Space systems are often SoS composed of satellites and other constituents in ground and space to accomplish missions related to environmental monitoring and data distribution, and telecommunication signal providing. In Brazil, the National Institute of Space Research (INPE, in Portuguese) is the entity dedicated to research and space exploration. They manage satellites and ground stations, and manage and distribute environmental data and satellite images. The launching of a satellite into space is costly to space systems. 
The launch of a CubeSat, an open source architecture of $10 \mathrm{~cm}$ X $10 \mathrm{~cm}$ X $10 \mathrm{~cm}$, for example, is estimated as US\$ 80,000. Due to such high costs and relevant potential losses, the system is considered as a critical domain, which justifies the use of M\&S techniques to avoid losses. In Brazil, the Space SoS is composed of the following different types of constituents:

Command and Control Center (C2): located in São José dos Campos, it is responsible for (i) sending telecommands (data upload to a satellite); (ii) telemetry reception (data download from the satellite); (iii) mission data reception; (iv) satellites tracking; (v) orbit propagation and determination; and (vi) flight plans generation.

Satellites: are responsible for capturing the images of territory. There are three satellites, two dedicated to data collection (SCD1 and SCD2) and one for remote sensing (CBERS 4A).

Ground Station: located in Cuiabá, it involves reception and satellite data transfer, and temporarily stores image data and satellites tracking;

Remote Sensing Data Center: receives records, processes, storage, and distributes images and data from remote sensing; and

Data Collection Platform (DCP): a ground device whose electronic sensors measure environmental variables such as precipitation, atmospheric pressure, solar radiation, temperature, air humidity, dew point, wind direction and speed, and detect variations in water bodies levels. They are spread in the entire Brazilian territory. When satellites passes over them, such DCP data are automatically transferred to artificial satellites in the Earth orbit and retransmitted to ground stations to be distributed to end users, thus enabling the monitoring of large territorial extensions and remote areas.

We have established a partnership with INPE and developed a M\&S solution based on our SosADL2DEVS transformation to model and simulate the Brazilian Space SoS. We aimed at investigating the results of the use of a satellite constellation, i.e., a set of satellites used in association for the improvement in the services provided (as telecommunications). This was justified because such constellation is to be soon launched to space, since BRICS space agencies - Brazil, Russia, India, China and South Africa - have discussed technical aspects related to the five countries' initiative in establishing a constellation of six (6) remote sensing satellites. Moreover, all the constituents were submitted to a resource competition, i.e., since constituents keep their operational independence and also contribute to several missions being carried out in a same SoS, in a given moment, a constituent (such as a satellite) can be required to perform a task of a mission (capture a picture of Amazon, for example), but it is also involved in the accomplishment of another mission (receiving data from DCP stations), evidencing a resource competition that requires management and scheduling, unless parallel processing is available.

Two missions were assigned to that SoS: (i) To perform the environmental data collection from DCP stations via satellites and provide that to the Ground station for distribution purposes; and (ii) To obtain pictures from the Amazon region using the satellite. We were also concerned to simulate the Space SoS with all DCP stations in Brazil, increasing the number of satellites from one to six to measure (i) the level of resource competition, the percentage with which the missions were met in relation to the expected, and (ii) how the increase in the number of satellites improves the performance of a SoS as a whole, reducing the waiting time to receive a requested data from the satellite.

We modeled a SoS architecture with a C2 center, a data center, a ground station, six satellites, and 249 DCP stations (a total of 258 constituents). The orbits were defined according to a study on the constellation of satellites (de Carvalho, dos Santos Lima, dos Santos Jotha, and de Aquino 2013). Maximum and average contact times, maximum and average revisit times, percentage of satisfactory revisits and the average number of contacts per day were observed during the preparation for simulation. The configuration chosen was three orbital planes with two satellites in each plane were adopted, as recommended by Carvalho et al. (de Carvalho, dos Santos Lima, dos Santos Jotha, and de Aquino 2013). 
We obtained data from INPE corresponding to a period between January 1st, 2017 and October 31st, 2017 from each DCP station in operation the entire Brazilian territory. The satellites used for the simulation were: a) Sino-Brazilian Satellite of Earth Resources (CBERS-4) (Period: 100.3 minutes); b) Data Collection Satellite 1 (SCD 1) (Period: 99.7 minutes), and c) Data Collection Satellite 2 (SCD 2) (Period: 99.7 minutes). A script was also developed for accessing an external link that provide these coordinates, which enabled a revisit period of 30 minutes. Each orbit contained two satellites with different movements that enable them to revisit the same DCP station each 30 minutes. We performed the model transformation, produced the DEVS models, deployed in MS4ME environment, and executed the simulation. The total simulation time was approximately 34 hours.

We concluded that, for all cases, an increase in the number of satellites reduced the competition between missions and DCP stations, and increased the number of data effectively transferred, justifying the acquisition of new satellites by the Brazilian government. Secondly, an increase in the number of satellites reduced data losses, as expected.

\section{DISCUSSION}

Modeling and simulation are distinct activities (Zeigler, Mittal, and Traore 2018). As a matter of fact, the languages used to capture each one of these branches are actually complementary since they have different levels of abstraction. At least for our context, we could conjecture that modeling addresses software engineering side, whilst simulation addresses systems engineering part. Regarding software engineering side, modeling is often concerned to capture systems using languages in high-level of abstraction, with not much details on hardware issues, and representing the relevant characteristics of a software as a valid simplification of the reality (Zeigler, Muzy, and Kofman 2019). However, we recognize that those languages still have some pitfalls particularly related to representation of dynamics of multiple systems interoperating and potentially changing at runtime. Moreover, those models are semi-formal, which means that guaranteeing their correctness or the resulting quality of the product constructed using them is often difficult and non-reliable. On the other hand, as corroborated by Zeigler, simulation deals with dynamics (Zeigler, Muzy, and Kofman 2019). Moreover, simulation models hold a mathematical foundation and semantics that enables a system engineer to model a system in an unambiguous format, which is also a valuable property for all types of engineering, including software engineering. However, exclusively using simulation models could be not productive for software engineers, since formalisms such as DEVS deal with behaviors expressed as state machines and input/output ports and stimuli, which can be hard to read, visualize, and maintain, specially if we consider complex behaviors and multiple systems. Zeigler states that model-based methods need to be augmented with simulation-based methodologies to ensure they support complex systems engineering that integrate discrete and continuous systems for complex hybrid systems (Zeigler, Mittal, and Traore 2018). Then the combination of both paradigms (modeling and simulation) offers a framework in high-level of abstraction for specification of SoS software architectures in addition to a formal foundation that allows formal verification of the system simulation models, claiming for an increase in the quality of the resulting system.

Another important issue on $\mathrm{M} \& \mathrm{~S}$ frameworks is the experimental frame. An experimental frame comprises the set of conditions under which system is observed or experimented with (Zeigler, Muzy, and Kofman 2019). Zeigler et al. mention, for illustration purposes, the simulation of fire in a forest. They say that, out of the multitude of variables that relate to a forest, the set lightning, rain, wind, smoke represents one particular choice. Such an experimental frame is then motivated by the interest in modeling the way lightning ignites a forest fire. They also mention that a more refined experimental frame would add the moisture content of the vegetation and the amount of unburned material as variables. In our context, from the multiple variables and perspectives that could be adopted to represent and simulate a Space SoS, our experimental frame comprised the involved relevant constituents to study how the increase in the number of satellites would affect the accomplishment of the missions. M\&S foundations also include other elements that should 
be used to make the experimental frame feasible and reliable, such as the generator, that generates input segments to the system; the acceptor that monitors an experiment to see the desired experimental conditions are met; and the transducer that observes and analyzes the system output segments (Zeigler, Muzy, and Kofman 2019). During our research, we actually provided equivalent entities for some of those elements, such as the stimuli generators to represent the M\&S generator; the software engineer, that monitors the simulation to observe if the desired behaviors emerge from the SoS (acceptor); and the simulation logs, that plays the transducer role and are used to be analyzed by a statistical model-checker.

The solutions reported here have been developed under the software engineering perspective. However, as have been recently reported (Zeigler, Mittal, and Traore 2018), both software and systems engineer face the same challenges regarding M\&S solutions for SoS. We believe we have progressed until the third level of maturity of those presumed by INCOSE MBSE Roadmap (Zeigler, Mittal, and Traore 2018). Under INCOSE perspective and correspondences to DEVS, we adopted DEVS model standard and DEVS simulation protocol standard (Level 1), employed Experimental Frame representation of metrics and DEVS models for Integrated Systems (Level 2), and adopted architecture models integrated with simulation, analysis, and visualization (Level 3). Then, from now on, we are at the same point seeking for advances to overcome the remaining challenges, which leads to the next section.

\subsection{Challenges}

SoS are inherent adaptive and dynamic since they interact and adapt to the dynamic environment (Zeigler, Mittal, and Traore 2018). We have progressed in many directions to provide a M\&S solution for Software Engineering of SoS. However, specific advances are still necessary to cope with important needs regarding $\mathrm{M} \& \mathrm{~S}$ for SoS. We discuss them, as follows.

Simulation of quality attributes. We have already achieved a reasonable simulation of some quality attributes of a SoS software architecture, including functional suitability (the degree to which a product or system provides functions that meet stated and implied user needs - missions or goals offered by a SoS at this context) and some level of availability and interoperability (ISO/IEC 2010). Security has also been a matter of investigation (Hachem, Chiprianov, Neto, and Aniorté 2018). However, precisely representing and simulating quality attributes require for reproducing each of the conditions that a system is subject to provide that attribute. Then, more detailed experimental frames are necessary for each of the prioritized quality attributes of a SoS software architecture. This is still matter of investigation for the next years.

Limited computational power. Zeigler et al. state that complex systems engineering is still in infancy as the tool-set support for engineering complex systems is limited. These limitations include a lack of a full-strength $M \& S$ computational substrate. In our studies, we conducted simulations that took more than 30 hours to simulate SoS with a couple hundreds of constituents using powerful processors (Graciano Neto, Manzano, Kassab, and Nakagawa 2018). Since a Smart city SoS can have millions of constituents, a linear relation claims for some years of simulation time and multiple powerful processors to simulate a smart city structure and behavior. Then, advances shall also be achieved regarding this topic.

Generalization of SoS simulation models. Under M\&S conceptual framework, modeling can be seen as a valid simplification of the reality, i.e., reduction of complexity to enable a model to be executed on resource-limited simulators within some experimental frame of interest (Zeigler, Mittal, and Traore 2018). Indeed, according to the framework, there is always at least one pair of models involved, which are called the base and lumped models, in such a relationship. A base model is a more generic one that represents the family of architectures that could be derived from a set of constituents that form a SoS; whilst a lumped model is one architectural instance derived from that base model. Simulators such as MS4ME 
provide an automatic solution for obtaining a lumped model from a base model, besides the adoption of an experimental frame. However, a simulation model still represents only a portion of the reality at small-scale (couple hundreds of potentially millions constituents of a real SoS). Then, advances on megamodels for SoS simulation could exploit the formal foundations of DEVS models and obtain, as a possibility, the validity of a model by mathematical induction mechanisms, for example.

Coverage of SoS simulation models. Multiple combinations of constituents and inputs/outputs are possible in a SoS. This leads to an essentially combinatorial (NP-Hard) problem. Then, there is a need to guarantee that there is coverage in a simulation model, i.e., the assumption that the simulation model is exhaustive enough to test the maximum possible adverse conditions to which a SoS and its constituents can be submitted in the real world. Advances in co-simulation, SoS software testing based on simulations, and experimental frames can alleviate this challenge.

\section{FINAL REMARKS}

This paper reported an industrial application of M\&S for a Space SoS conducted as a joint effort between INPE and researchers from four universities. Simulation models specified in DEVS were automatically produced from a model transformation. The results yielded by the simulation confirmed that the acquisition of new satellites can improve the services provided by the Brazilian Space SoS, particularly in regards to environmental data distribution, Amazon monitoring. Besides obtaining important conclusions from the experiment drawn using the M\&S framework, that experience enabled us to glimpse important advances that must still be achieved to guarantee a higher quality for SoS to be deployed in the real world.

We also discussed important contributions achieved, the alignment with the level of maturities of INCOSE Roadmap, the equivalence of some concepts we have worked on, the common problems that software and systems engineering communities share regarding engineering of complex adaptive SoS, and challenges that still remain for both. We expect this experience report can foster discussions and work as a north for discussion and advances in the state of the art and practice in industrial solutions of M\&S for Software and Systems engineering of SoS.

\section{ACKNOWLEDGMENTS}

Wallace Manzano and Elisa Yumi Nakagawa thank São Paulo Research Foundation (FAPESP) (grants: 2017/06195-9, 2017/17448-5, and 2018/21517-5).

\section{REFERENCES}

Bass, L., P. Clements, and R. Kazman. 2012. Software Architecture in Practice. 3rd ed. Indianapolis, Indiana, USA, Addison-Wesley Professional.

Blair, G., N. Bencomo, and R. France. 2009, Oct. "Models at run.time". Computer vol. 42 (10), pp. 22-27.

Boardman, J., and B. Sauser. 2006. "System of Systems - the meaning of 'of"”. In 1st SoSE, pp. 1-6. Los Angeles, USA, IEEE.

Bogado, V., S. Gonnet, and H. Leone. 2014. "Modeling and Simulation of Software Architecture in Discrete Event System Specification for Quality Evaluation”. Simulation vol. 90 (3), pp. 290-319.

Brazilian Space Agency 2018. "PNAE - National Program of Space Activities 2012-2021". Available at: http://www.aeb.gov.br/central-de-conteudos/publicacoes/pnae Access: December 2018.

Cavalcante, E., N. Cacho, F. Lopes, and T. Batista. 2017. "Challenges to the Development of Smart City Systems: A System-of-Systems View”. In 31st SBES, pp. 244-249. Fortaleza, CE, Brazil, ACM. 
de Carvalho, M. J. M., J. S. dos Santos Lima, L. dos Santos Jotha, and P. S. de Aquino. 2013. "CONASAT Constellation of Nano Satellites for Environmental Data Collection (In portuguese)". In 16th Brazilian Symposium on Remote Sensing, pp. 9108-9115. Foz do Iguaçu, Brazil, INPE.

Dobrica, L., and E. Niemele. 2002, July. "A Survey on Software Architecture Analysis Methods". IEEE Transactions on Software Engineering vol. 28 (7), pp. 638-653.

Garlan, D., F. Bachmann, J. Ivers, J. Stafford, L. Bass, P. Clements, and P. Merson. 2010. Documenting Software Architectures: Views and Beyond. 2nd ed. Westfold, Massachussets, Addison-Wesley.

Goncalves, M. B., E. Cavalcante, T. Batista, F. Oquendo, and E. Y. Nakagawa. 2014. "Towards a conceptual model for Software-intensive System-of-Systems". In SMC, pp. 1605-1610. San Diego, USA, IEEE.

Graciano Neto, V. V. 2018, March. A simulation-driven model-based approach for designing softwareintensive systems-of-systems architectures. Ph. D. thesis, ICMC - USP.

Graciano Neto, V. V., L. Garcés, M. Guessi, C. Paes, W. Manzano, F. Oquendo, and E. Y. Nakagawa. 2018. "ASAS: An Approach to Support Simulation of Smart Systems". In 51st HICSS 2018, pp. 5777-5786. Big Island, Hawaii, USA, IEEE.

Graciano Neto, V. V., M. Guessi, L. B. R. Oliveira, F. Oquendo, and E. Y. Nakagawa. 2014. "Investigating the Model-Driven Development for Systems-of-Systems". In 8th ECSAW, pp. 22:1-22:8. Vienna, Austria, ACM.

Graciano Neto, V. V., W. Manzano, L. Garcés, M. Guessi, B. Oliveira, T. Volpato, and E. Y. Nakagawa. 2018. "Back-SoS: Towards a Model-based Approach to Address Architectural Drift in Systems-of-Systems". In The 33rd ACM/SIGAPP Symposium On Applied Computing (SAC 2018), pp. 1-3. Pau, France, ACM.

Graciano Neto, V. V., W. Manzano, M. Kassab, and E. Y. Nakagawa. 2018. "Model-based Engineering \& Simulation of Software-intensive Systems-of-systems: Experience Report and Lessons Learned". 12th ECSA (Companion), pp. 27:1-27:7. Madrid, Spain, ACM.

Graciano Neto, V. V., C. E. Paes, L. Garcés, M. Guessi, F. Oquendo, and E. Y. Nakagawa. 2017. "StimuliSoS: A Model-Based Approach to Derive Stimuli Generators in Simulations of Software Architectures of Systems-of-Systems". Journal of the Brazilian Computer Society vol. 23 (1), pp. 13:1-13:22.

Guessi, M., E. Cavalcante, and L. B. R. Oliveira. 2015, May. "Characterizing Architecture Description Languages for Software-Intensive Systems-of-Systems”. In 3rd SESoS, pp. 12-18. Florence, Italy, IEEE.

Guessi, M., V. V. Graciano Neto, T. Bianchi, K. R. Felizardo, F. Oquendo, and E. Y. Nakagawa. 2015, April. "A systematic literature review on the description of software architectures for systems of systems". In 30th SAC, pp. 1433-1440. Salamanca, Spain, ACM.

Hachem, J. E., V. Chiprianov, V. V. G. Neto, and P. Aniorté. 2018. "Extending a Multi-Agent Systems Simulation Architecture for Systems-of-Systems Security Analysis”. In 13th SoSE, pp. 276-283. Paris, France.

INCOSE 2018. "The Guide to the Systems Engineering Body of Knowledge (SEBoK)". Technical Report SEBoK1.9.1, International Council on Systems Engineering (INCOSE), San Diego, CA.

ISO 2011, Dec. "ISO/IEC/IEEE Systems and software engineering - Architecture description". ISO/IEC/IEEE 42010:2011, pp. 1-46.

ISO/IEC 2010. "ISO/IEC 25010 System and software quality models". Technical report.

Klein, J., and H. van Vliet. 2013. "A Systematic Review of System-of-systems Architecture Research". In 9th QoSA, pp. 13-22. Vancouver, Canada, ACM.

Lana, C. A., N. M. Souza, M. E. Delamaro, E. Y. Nakagawa, F. Oquendo, and J. C. Maldonado. 2016, October. "Systems-of-Systems Development: Initiatives, Trends, and Challenges". In 42nd CLEI 2016, pp. 1-10. Valparaiso, Chile, IEEE. 
Maier, M. W. 1998. "Architecting principles for systems-of-systems". Systems Engineering vol. 1 (4), pp. 267-284.

Manzano, W., V. V. Graciano Neto, and E. Y. Nakagawa. 2018. "Dynamic-SoS: An Approach for the Simulation of System-of-Systems Dynamic Architectures". The Computer Journal vol. X (Y), pp. 1 - 16. Accepted for Publication.

Medvidovic, N., and R. N. Taylor. 2000, January. "A Classification and Comparison Framework for Software Architecture Description Languages”. IEEE Trans. Softw. Eng. vol. 26 (1), pp. 70-93.

Michael, J. B., R. Riehle, and M. T. Shing. 2009, May. "The verification and validation of software architecture for systems of systems". In 4th IEEE International Conference on System of Systems Engineering (SoSE 2009), pp. 1-6. Albuquerque, USA, IEEE.

Nielsen, C., P. Larsen, J. Fitzgerald, J. Woodcock, and J. Peleska. 2015. "Systems of systems engineering: Basic concepts, model-based techniques, and research directions". ACM Computing Surveys vol. 48 (2).

OMG 2015. "UML: Unified Modeling Language". Available at: http://www.omg.org/spec/UML/ About-UML/. Access: January 2018.

OMG 2019. "The OMG Systems Modeling Language (SySML) Version 1.5". Available at: http://www.omgsysml.org/. Last Access: February 2019.

Oquendo, F. 2016a, June. "Formally Describing the Software Architecture of Systems-of-Systems with SosADL". In 11th IEEE SoSE, pp. 1-6. Kongsberg, Norway, IEEE.

Oquendo, F. 2016b, June. " $\pi$-Calculus for SoS: A Foundation for Formally Describing Software-intensive Systems-of-Systems". In 11th IEEE SoSE, pp. 1-6. Kongsberg, Norway, IEEE.

Oquendo, F. 2016c. "Software Architecture Challenges and Emerging Research in Software-Intensive Systems-of-Systems". In 10th ECSA, pp. 3-21. Copenhagen, Denmark, Springer.

Piccolboni, L., and G. Pravadelli. 2014. "Simplified stimuli generation for scenario and assertion based verification". In 15th LATW, pp. 1-6. Fortaleza, Brazil, IEEE.

Rajhans, A., A. Bhave, S. Loos, B. H. Krogh, A. Platzer, and D. Garlan. 2011, Dec. "Using parameters in architectural views to support heterogeneous design and verification". In 50th CDC, pp. 2705-2710, IEEE.

Rajhans, A., A. Bhave, I. Ruchkin, B. H. Krogh, D. Garlan, A. Platzer, and B. R. Schmerl. 2014. "Supporting Heterogeneity in Cyber-Physical Systems Architectures". IEEE Trans. Automat. Contr. vol. 59 (12), pp. 3178-3193.

Shea, Garrett 2017. "NASA Systems Engineering Handbook Revision 2".

Taylor, R. N., N. Medvidovic, and E. M. Dashofy. 2010. Software Architecture - Foundations, Theory, and Practice. Hoboken, New Jersey, USA, Wiley.

Yang, S., R. Wille, D. Grobe, and R. Drechler. 2012. “Coverage-Driven Stimuli Generation”. In 15th Euromicro Conference on Digital System Design (ECDSD 2012), pp. 525-528. Izmir, Turkey, IEEE.

Yingchao, Z. 2012, July. "System of Systems complexity and decision making". In 2012 7th International Conference on System of Systems Engineering (SoSE), pp. 509-513.

Zeigler, B. P., S. Mittal, and M. K. Traore. 2018. "MBSE with/out Simulation: State of the Art and Way Forward". Systems vol. 6 (4).

Zeigler, B. P., A. Muzy, and E. Kofman. 2019. Theory of Modeling and Simulation. 3nd ed. Orlando, FL, USA, Academic Press, Inc.

Zeigler, B. P., H. S. Sarjoughian, R. Duboz, and J.-C. Souli. 2012. Guide to Modeling and Simulation of Systems of Systems. London, United Kingdom, Springer-Verlag London. 


\section{AUTHOR BIOGRAPHIES}

VALDEMAR VICENTE GRACIANO-NETO received his Ph.D. degree in computer science and computational mathematics from the Institute of Mathematical Sciences and Computation, University of São Paulo, Brazil and the Docteur degree in sciences and information technology from Université Bretagne-Sud, Lorient, France, in 2018. He is an Assistant Professor with the Institute of Informatics, Federal University of Goiás, Goiânia, Brazil, and also the Coordinator of the Special Committee on Information Systems of the Brazilian Computer Society (SBC) in the 2018-2019 period. Dr. Graciano Neto is a member of the SBC and the ACM. His email address is valdemarneto@inf.ufg.br.

CARLOS EDUARDO PAES received the Ph.D. degree in computer science from the Technological Institute of Aeronautics, São José dos Campos, Brazil, in 2008. He conducted his postdoctoral in systemsof-systems engineering in 2015-2017 with the Department of Computer Systems, University of São Paulo - USP, São Paulo, Brazil. In 2015-2016, he conducted research internship with the University of South Brittany, Morbihan, France. He participated in projects in defense and air traffic control in Brazil. He is a Professor with the Department of Computer Science, Pontifical Catholic University of São Paulo, São Paulo, Brazil. Dr. Paes is also a member of the Brazilian Computer Society (SBC). His email address is carlosp@pucsp.br.

ADAIR JOSE ROHLING received his Ph.D. degree in Space Systems Engineering and Management from INPE, the Brazilian National Institute of Space Research. He is an Adjunct Professor at the Federal Technological University of Paraná, in Guarapuava, Brazil. His email address is adairrohling@utfpr.edu.br.

WALLACE MANZANO is an Information Systems degree undergraduate student at University of São Paulo, in São Carlos, Brazil. He has experience and has investigated systems-of-systems, model-driven development, software architecture, and simulation models during the last three years. His email address is wallace.manzano@usp.br.

ELISA YUMI NAKAGAWA is an associate professor in the Department of Computer Systems at the University of São Paulo - USP, Brazil. She conducted her post-doctoral research at Fraunhofer IESE, Germany, in 2011-2012 and at the University of South Brittany, France, in 2014-2015. She received her Ph.D. degree from USP in 2006. She coordinates international/national research projects in software architecture, reference architectures, and systems-f-systems, and also supervises PhD/Master's students and post-doctoral researchers. She has organized international/national conferences and has served as a program committee member at many conferences and as a reviewer of various journals. She is a member of IEEE and SBC (Brazilian Computer Society). Her email address is elisa@icmc.usp.br. 